\title{
Baroclinic Instability of Buoyancy-Driven Coastal Density Currents
}

\author{
Bo QIU AND NoRIHISA IMASATo \\ Geophysical Institute, Kyoto University, Kyoto, Japan
}

\author{
TOSHIYUKI AWAJI
}

Department of Applied Science, Kochi Women's University, Kochi, Japan

\begin{abstract}
Coastal density currents induced by buoyancy supply along an upper coast are studied in a threedimensional numerical model. Scaling analysis of the governing equations reveals that when the vertical eddy diffusivity is small, the horizontal Ekman number $E_{h}=A_{h} f /\left(g^{\prime} Q_{e}\right)^{2 / 3}$ is the crucial parameter determining the structures of the current, where $A_{h}$ is the horizontal eddy viscosity, $Q_{e}$ is the discharge rate per unit length along the coast, $g^{\prime}$ is the reduced gravity constant, and $f$ is the Coriolis parameter. Based on numerical experiments with $A_{h}$ and $Q_{e}$ varied as external parameters, a critical $E_{h}\left(E_{h c}\right)$ is found between 0.354 and 0.566 . The density current develops unstably below this value. In cases where $E_{h}<$ $E_{h c}$, excessive accumulation of fresh water causes sudden density changes near the coast, which eventually breaks down the quasi-steady balance of the current and triggers small-scale disturbances in the longshore direction. The large-scale unstable waves induced by these disturbances have length scales of the order of $100 \mathrm{~km}$ and an $e$-folding time of several days. Study of the energetics revealed that the unstable waves grow via the baroclinic process, and as they develop into the finite-amplitude stage, an energy transfer from the eddy field to the mean field accelerates the density current near the coast and induces an oppositely flowing mean flow offshore. These induced flows are important in the detachment process of the fully developed unstable eddies.
\end{abstract}

\section{INTRODUCTION}

Horizontal density differences between light coastal water and saltier offshore water tend to produce coastal density currents moving with the coast to the right (left) in the northern (southern) hemisphere. Both field observations and recent satellite pictures show that many of such coastal currents are highly unstable. For example, Figure 1 shows a thermal infrared picture of the East China Sea taken by the NOAA 9 satellite on March 24, 1986. In this picture the lighter tones represent colder water from the coast, and the darker tones represent warmer offshore water. Large-amplitude wave patterns with a length scale of $50 \mathrm{~km}$ can be easily seen on the density current, which flows southerly along the east coast of China and is induced by the freshwater runoff from the Yangtze and other rivers. Similarly, Zhang and Klemas [1982] found from satellite pictures an elliptic cyclonic ring with a long axis of $120 \mathrm{~km}$ and a short axis of $60 \mathrm{~km}$ and some other smaller cyclonic rings on this density current.

Like on the density current of the East China Sea, seaward projections of lighter water and unstable waves were detected on the Leeuwin Current [Legeckis and Cresswell, 1981 ; Cresswell and Golding, 1980], the Norwegian Coastal Current [Mork, 1981] and on the Ligurian Coastal Current [Crepon et al., 1982]. It is noteworthy that all the unstable waves and eddies observed on the coastal density currents have a length scale of 50 to $100 \mathrm{~km}$ and have a large amplitude. This latter fact implies that the underlying dynamics of the unstable waves are strongly nonlinear.

The unstable behavior of the coastal density currents has prompted several laboratory and theoretical investigations. By releasing buoyant fluid from the inner circular wall of a rotat-

Copyright 1988 by the American Geophysical Union.

Paper number $8 \mathrm{C} 0062$.

0148-0227/88/008C-0062\$05.00 ing cylinder, Griffiths and Linden [1981, 1982] showed that wavelike perturbations develop on the quasi-steady azimuthal flow near the wall as it develops in width. The waves then grow in amplitude, break on the upstream side, and eventually distort the flow pattern. By releasing buoyant water through a point source, Stern et al. [1982] and Griffiths and Hopfinger [1983] also showed experimentally that the coastal density currents are unstable turbulent flows.

Under the assumption of the uniform potential vorticity of the flow, Stern [1980] was the first to theoretically find the solutions that represent breaking waves, blocking waves, and cyclone-anticyclone vortex pairs on the density flow. Using the reduced gravity model, instability on coastal density currents was further studied by Killworth and Stern [1982], Paldor [1983], Kubokawa and Hanawa [1984] and Kubokawa [1986]. It was found that a density current may become unstable if the potential vorticity of the current is not uniform. In the case of uniform potential vorticity, the density current is unstable only when the frontal trapped and coastal trapped waves coalesce [Kubokawa, 1986].

Concerning the behavior of large-amplitude unstable waves on density flows, Stern [1985] and Pratt and Stern [1986] investigated a piecewise uniform potential vorticity flow using the contour dynamics method. They studied the influence of initial disturbance conditions on the shingle formation and on eddy detachment from the basic flow and obtained similar wave patterns between the numerical result and the observation from the Gulf Stream surface front [e.g., Lee and Atkinson, 1983]. Since their studies concerned the Gulf Stream frontal system, the model was also based on the one-layer reduced gravity model, which neglects motion in the lower layer. In the real coastal areas, however, the lower layer is generally shallow. As was noted by Killworth et al. [1984] in a two-layer model, an active lower layer may cause new types of instability (baroclinic instability) on the density front. Thus it is necessary to take the motion in the shallow lower layer into 


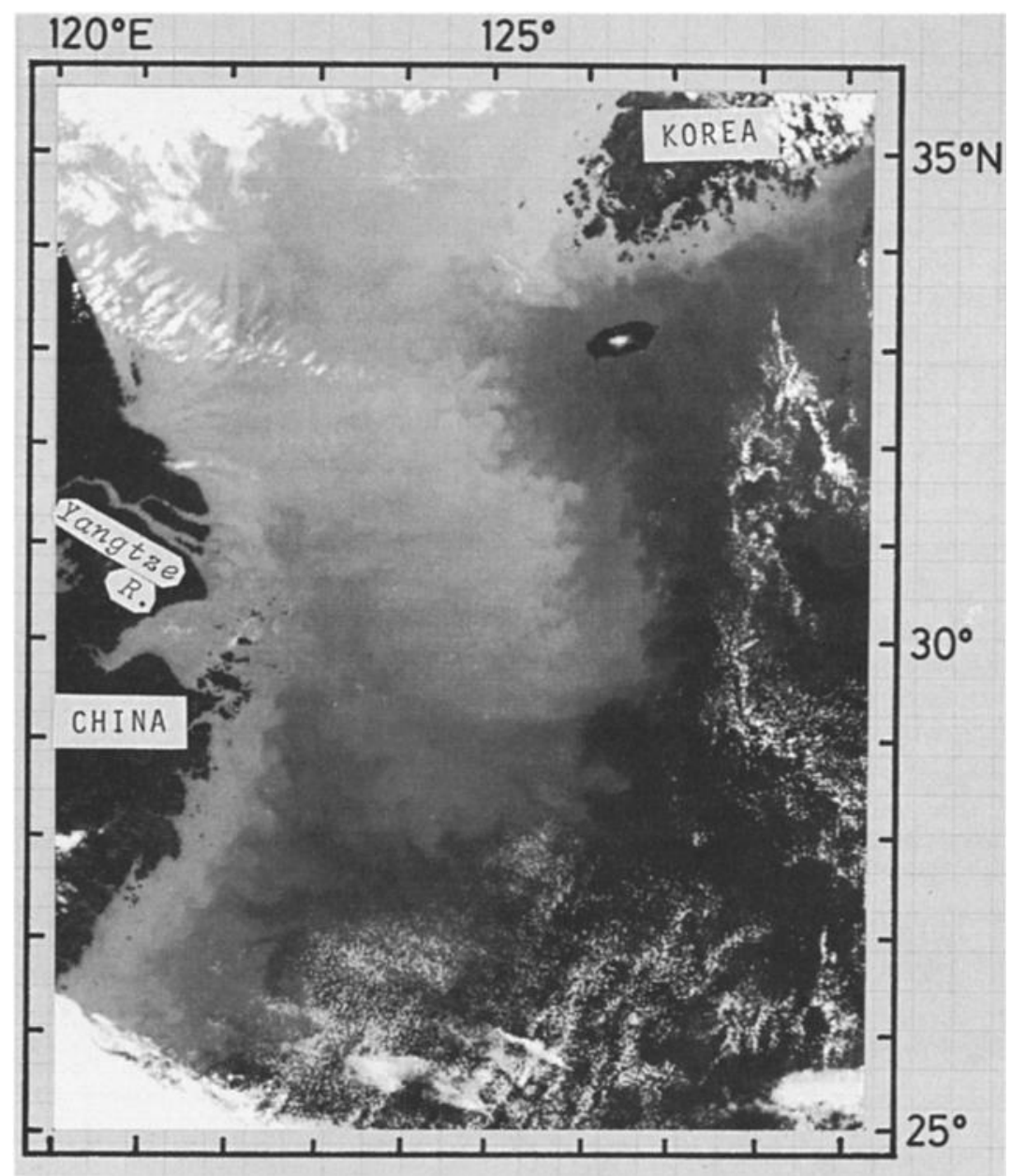

Fig. 1. Thermal infrared picture of the East China Sea taken from the NOAA 9 satellite on March 24, 1986.

account when investigating the instability of coastal density currents.

In this study we investigate the instability of coastal density currents using a three-dimensional primitive equation model in a periodic channel basin. Since the main portion of density currents generally extends over the flat continental shelf, we replace the narrow bottom slope near the coast by a vertical coastal wall and assume the basin's bottom to be flat. We produce the density currents in the model basin by introducing buoyancy flux constantly and uniformly from the upper coastal wall. In real coastal areas, buoyancy supply varies in time as well as in the longshore direction. These variations, however, can be neglected if we are to study the fundamental processes associated with the unstable density currents.

Special attention in the present study is directed to the occurrence of initial disturbances, the energetics of the unstable waves, and the eddy mean flow interactions after the unstable waves develop into the finite-amplitude stage. The eddy detachment in the finite-amplitude stage of the unstable waves is important in water mass exchanges across the density currents. This detachment process is investigated in the present study using the technique of Lagrangian particle tracking [Awaji et al., 1980; Imasato et al., 1980]. Unlike other methods [e.g., Stern, 1985], this tracking method enables us to track water movement even after eddies detach from their original water mass. In shallow coastal areas, turbulence is easily generated by meteorological disturbances or strong tidal currents. These factors are regarded as subgrid-scale phe- nomena in the present study and are introduced into the model in the form of eddy diffusivity. How changes in the eddy diffusivity and in the buoyancy supply rate may influence the criterion of density currents' instability is also investigated.

In the next section we describe the model's system and select external parameters for the numerical calculations. In section 3, characteristics of a representative unstable density current are investigated in detail. The energetics involved in the unstable processes are further studied in section 4 . In section 5 we discuss the dependence of unstable currents on the external parameters and compare the result with those derived from a two-layer frictional quasi-geostrophic model. In the last section the results are summarized and their applications to the real coastal currents are discussed.

\section{Model Description}

Under the Boussinesq and hydrostatic approximations, the model ocean on the $f$ plane is governed by following equations:

$$
\begin{gathered}
u_{t}+L(u)-f v=-P_{x} / \rho_{0}+A_{v} u_{z z}+A_{h}\left(u_{x x}+u_{y y}\right) \\
v_{t}+L(v)+f u=-P_{y} / \rho_{0}+A_{v} v_{z z}+A_{h}\left(v_{x x}+v_{y y}\right) \\
P_{z}=-g \rho \\
u_{x}+v_{y}+w_{z}=0 \\
S_{t}+L(S)=K_{v} S_{z z}+K_{h}\left(S_{x x}+S_{y y}\right) \\
\rho=\rho_{0}(1+\beta S)
\end{gathered}
$$


where $(u, v, w)$ are velocities in the $(x, y, z)$ directions, $\rho$ is the density, $\rho_{0}$ is a reference density, $S$ is the salinity, and $f$ is a constant Coriolis parameter. The subscripts $x, y, z$, and $t$ denote partial differentiation, and the advective operator $L$ is given by $L(\sigma)=(u \sigma)_{x}+(v \sigma)_{y}+(w \sigma)_{z} . A_{v}$ and $A_{h}$ are coefficients of the vertical and horizontal eddy viscosity, respectively. $K_{v}$ and $K_{h}$ are eddy diffusivities and are assumed to be equal to $A_{v}$ and $A_{h}$, respectively. For simplicity, only the linear effect of salinity on density is taken into account, such as in (6), where $\beta$ is the expansion coefficient for salinity.

The above governing equations are solved in the model basin shown in Figure 2, which is $240 \mathrm{~km} \times 800 \mathrm{~km}$ in size with a constant depth of $H_{0}=200 \mathrm{~m}$. In the model basin the $x$ axis is positive to the right along the coast, the $y$ axis is positive offshore, and the $z$ axis is positive upwards. Periodicity is assumed in the $x$ direction. Boundary conditions for the velocity field are no slip at the coast and free slip at the offshore wall, so that

$$
\begin{array}{cc}
u=v=0 & y=0 \mathrm{~km} \\
u_{y}=v=0 & y=240 \mathrm{~km}
\end{array}
$$

The model's surface and bottom are assumed to be free from fluxes of momentum, so that

$$
u_{z}=v_{z}=0 \quad z=0,-H_{0}
$$

At the surface a rigid-lid approximation is also adopted so as to eliminate the surface gravity waves for a more efficient calculation.

Fresh water is supplied uniformly along the upper coast (75 $\mathrm{m}$ deep from the surface as shown in Figure 2). Only buoyancy fluxes from freshwater discharge are introduced in this study, because the effect caused by mass fluxes can be neglected if the phenomena we are interested in have their length scale of internal Rossby radius and their time scale less than the inertial period [Ikeda, 1984]. The conversion from the amount of fresh water supplied to the positive buoyancy flux $B F$ can be expressed as

$$
B F=\rho_{0} \beta\left(S_{c}-S_{f}\right) Q_{e}
$$

where $Q_{e}$ is the discharge rate of fresh water per unit length along the coast, and $S_{f}$ and $S_{c}$ are the salinity values of the fresh water and the water adjacent to the coast, respectively. In the present study, $Q_{e}$ is fixed at a constant rate throughout the calculation, and no buoyancy fluxes are allowed to enter through the model's other surfaces. The initial state of the model is that the ocean basin is filled with homogeneous seawater of $S_{c}=34 \%$.

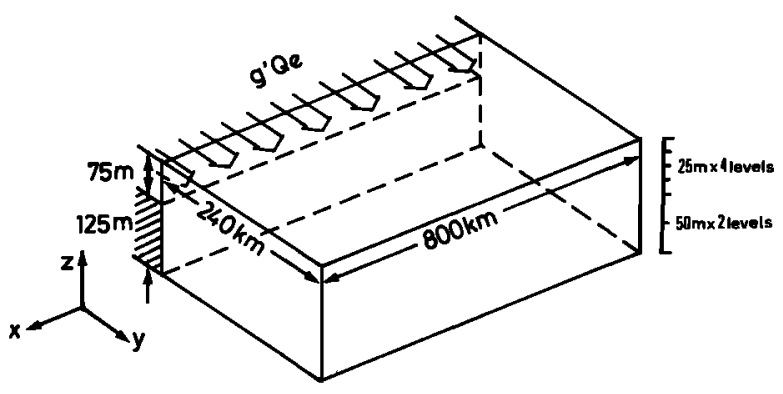

Fig. 2. The configuration of the model basin used in the numerical experiments. Buoyancy fluxes are supplied uniformly along the 75-m-deep upper coast.
TABLE 1. Different Combinations of $A_{h}$ and $Q_{e}$ Used in the Numerical Experiments

\begin{tabular}{ll}
\hline$Q_{e}, \mathrm{~cm}^{2} \mathrm{~s}^{-1}$ & \multicolumn{1}{c}{$A_{h}, 10^{7} \mathrm{~cm}^{2} \mathrm{~s}^{-1}$} \\
\hline 1500 & $0.08,0.1,0.2,0.35,0.5,0.8,1.0,1.3,3.0$ \\
3750 & $0.2,0.5,0.9,1.4$ \\
5250 & $0.6,1.1,1.9$ \\
\hline
\end{tabular}

If the freshwater supply is uniform in the longshore direction, the induced density current will grow independently of the $x$ direction before instability can occur. This indicates that the velocity and salinity profiles in the cross-shore $(y-z)$ section are essential in determining the occurrence and development of the initial disturbances. In the following we examine what external parameters determine the cross-shore section profiles of the density current. After assuming that $x$ derivatives are zero in the governing equations, and cross differentiating (3) and (2) with respect to $y$ and $z$ to eliminate the pressure, (1)-(5) reduce to

$$
\begin{gathered}
u_{t}+(v u)_{y}+(w u)_{z}-f v=A_{v} u_{z z}+A_{h} u_{y y} \\
\zeta_{t}+(v \zeta)_{y}+(w \zeta)_{z}-f u_{z}=-g \beta S_{y}+A_{v} \xi_{z z}+A_{h} \xi_{y y} \\
v_{y}+w_{z}=0 \\
S_{t}+(v S)_{y}+(w S)_{z}=A_{v} S_{z z}+A_{h} S_{y y}
\end{gathered}
$$

where $\zeta=-v_{z}$ is the $x$ component of relative vorticity. In order to nondimensionalize $(10)-(13)$, we introduce a time scale $T=1 / f$ and a horizontal velocity scale $U=\left(g^{\prime} Q_{e}\right)^{1 / 3}$, where $g^{\prime}=\rho_{0} \beta\left(S_{c}-S_{f}\right) g$ is the reduced gravity and $U$ corresponds to the buoyancy velocity defined by $K a o[1980,1981]$. We also introduce $L=U / f$ as a horizontal length scale, the basin depth $H_{0}$ as a vertical length scale, and a stretched salinity value $\tilde{S}=g \beta H_{0} S_{c} / f U L$ as a salinity scale. Based on these nondimensional variables

$$
\begin{gathered}
t^{*}=t / T \quad\left(x^{*}, y^{*}, z^{*}\right)=\left(x / L, y / L, z / H_{0}\right) \\
\left(u^{*}, v^{*}, w^{*}\right)=\left(u / U, v / U, w L / U H_{0}\right) \quad S^{*}=S / \tilde{S}
\end{gathered}
$$

equations (10)-(13) can be rewritten as

$$
\begin{gathered}
u_{t}+(v u)_{y}+(w u)_{z}-v=E_{v} u_{z z}+E_{h} u_{y y} \\
\zeta_{t}+(v \zeta)_{y}+(w \zeta)_{z}-u_{z}=-S_{y}+E_{v} \zeta_{z z}+E_{h} \zeta_{y y} \\
v_{y}+w_{z}=0 \\
S_{f}+(v S)_{y}+(w S)_{z}=E_{v} S_{z z}+E_{h} S_{y y}
\end{gathered}
$$

where asterisks indicating nondimensional variables are dropped. From (15)-(18), we find that the vertical Ekman number $E_{v}=A_{v} / f H_{0}{ }^{2}$ and the horizontal Ekman number $E_{h}=A_{h} / f L^{2}=A_{h} f /\left(g^{\prime} Q_{e}\right)^{2 / 3}$ are the two crucial external parameters which determine the cross-shore structures of the buoyancy-driven density current before the onset of instabilities.

In the following numerical experiments, we assume that the vertical dissipation effect is smaller than its horizontal counterpart and focus our discussion on how the horizontal Ekman number $E_{h}$ influences the instability of the coastal currents. In order to study this $E_{h}$ dependency, we use various combinations of $A_{h}$ and $Q_{e}$ in the numerical experiments (compare Table 1), while keeping other parameters fixed as follows: $f=6.84 \times 10^{-5} \mathrm{~s}^{-1}$ (referred at $28^{\circ} \mathrm{N}$ ), $S_{f}=0, \beta=$ $7.6 \times 10^{-4}$, and $A_{v}=1 \mathrm{~cm}^{2} \mathrm{~s}^{-1}$. The numerical method used 
(a) at $12.5 \mathrm{~m}$

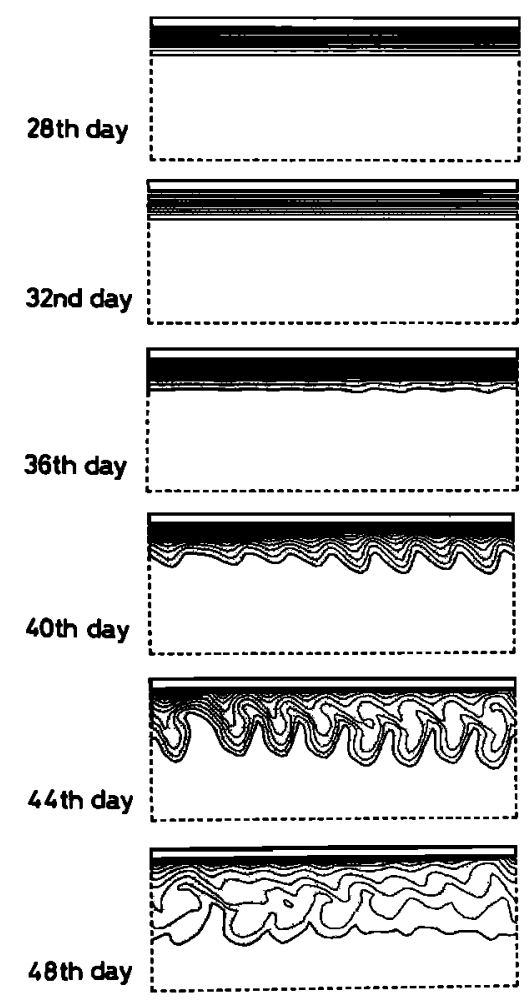

(b) at $87.5 \mathrm{~m}$
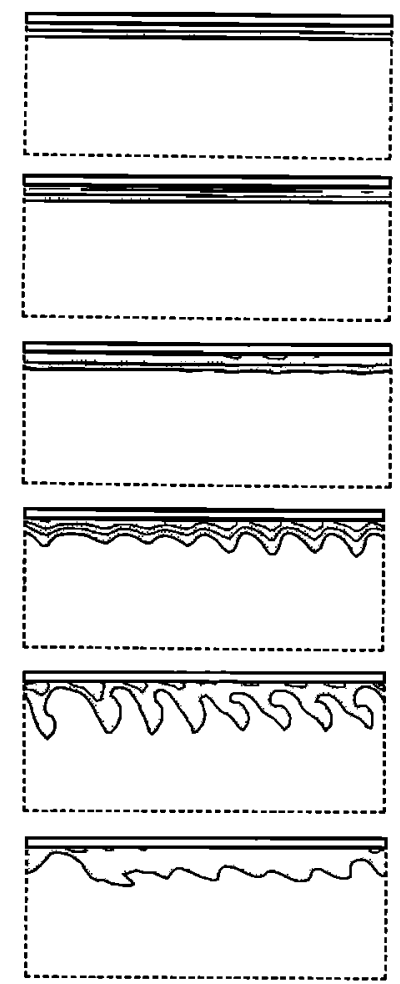

Fig. 3. The evolution of horizontal salinity distributions at depths of $(a) 12.5 \mathrm{~m}$ and $(b) 87.5 \mathrm{~m}$ for the representative unstable case. The contour value interval is $0.5 \%$ and salinity value in the unshaded outer ocean is $34.0 \%$.

in the present study is basicly that described by Semtner [1974] with some minor changes. The horizontal grid sizes are $\Delta x=10 \mathrm{~km}$ and $\Delta y=8 \mathrm{~km}$, which are fine enough to resolve wave patterns with wave lengths of $100-200 \mathrm{~km}$. In the vertical, six levels are chosen as shown in Figure 2.

\section{Behavior of the Unstable Density Current}

In this and the next section, a representative case in which the buoyancy-driven density current develops unstably is studied in detail. In this representative case, $A_{h}=1 \times 10^{6} \mathrm{~cm}^{2} \mathrm{~s}^{-1}$ and $Q_{e}=1500 \mathrm{~cm}^{2} \mathrm{~s}^{-1}$ (i.e., $E_{h}=0.071$ ). More general features of the density currents and their dependence on the horizontal Ekman number will be discussed in section 5 .

Figure 3 shows the time evolution of the horizontal salinity patterns for the representative case at two depth levels $(12.5 \mathrm{~m}$ and $87.5 \mathrm{~m}$ ). At the initial stage the density current grows uniformly along the coast, and its longshore velocity generally satisfies the thermal wind balance, $f u_{z}=g \beta S_{y}$. The density current's isohalines start to exhibit visible grid-scale disturbances in the longshore direction from day 30 . These disturbances then evolve rapidly into the large-scale unstable waves with a wavelength of $100 \mathrm{~km}$. The unstable wave crests on day 40 are found to lean backward in relation to the direction of the moving current. The existence of such "backward breaking waves" was also exhibited in a set of laboratory experiments by Griffiths and Linden [1981, 1982]. The amplitude of the unstable waves reaches its maximum value around day 44; thereafter wave patterns become obscure, and the horizontal salinity gradients weaken.

The growth of the unstable waves is also well manifested in Figure 4 where the temporal evolution of $v^{\prime}$ patterns is shown.
In this study an overbar denotes a zonal-averaged quantity and a prime denotes an eddy quantity which is the deviation from its zonal-averaged value, that is,

$$
\begin{gathered}
\bar{v}(y, z, t)=\frac{1}{L} \int_{0}^{L} v(x, y, z, t) d x \\
v^{\prime}(x, y, z, t)=v(x, y, z, t)-\bar{v}(y, z, t)
\end{gathered}
$$

where $L$ is the length of the basin. Two facts in Figure 4 are noteworthy. The first fact is that the phase of the unstable waves deflects to the $-x$ (upstream) direction as $z$ increases (compare the arrows on day 40 ). Since the vertical shear of the density current $\bar{u}_{z}$ is positive (from the thermal wind relation), it can be expected that the instability of the density current occurs via a baroclinic process [Pedlosky, 1979]. In other words, there is a transfer from the potential energy of the current to the eddy kinetic energy of the unstable waves. This expectation will be verified in the next section when we consider the energetics of unstable waves. The second fact is that as the unstable waves grow in amplitude, the patterns of $v^{\prime}$ elongate in the cross-shore direction and eventually split into two detached eddies (compare the patterns on day 46 in Figure 4).

In order to examine the detaching process of the eddies, we use the Euler-Lagrangian technique of tracking dynamically passive particles [Awaji et al., 1980; Imasato et al., 1980]. Since vertical motion in the present case is much weaker than horizontal motion, we confine our tracking particles to the surface level. The particles are initially distributed uniformly in the basin's surface layer. Figure 5 shows a sequence of tracking results beginning on day 24 (only the particles ap- 

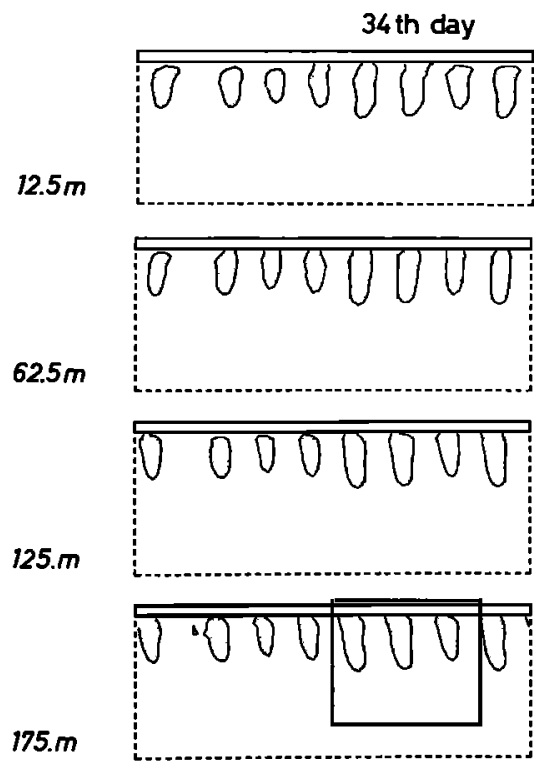

Fig. 4. The evolution of perturbed cross-shore velocity $v^{\prime}$ at depths of $12.5 \mathrm{~m}, 62.5 \mathrm{~m}, 125 \mathrm{~m}$ and $175 \mathrm{~m}$. The shaded areas indicate positive values. The vertical phase of the unstable waves leans to the $-x$ direction as shown by the arrows on day 40 . The contour value interval is $6.25 \mathrm{~cm} \mathrm{~s}^{-1}$.

pearing inside the dotted window of Figure 4 are depicted). As is indicated by the pattern on day 24 , light and dark particles are released initially inside and outside the density current, respectively. After instability occurs on the density current, we find that the current water moves rapidly offshore owing to the growth of the anticyclonic eddies (which correspond to the wave crests in Figure 3). Behind the anticyclonic eddies, the cyclonic eddies are found to effectively entrain the outer ocean water toward the coast. It is important to note that after the unstable waves decline from day 44 , the cyclonic eddies' entrainment of the outer ocean water weakens, and the eddies start to move downstream ( $+x$ direction) at a speed of $10 \mathrm{~cm}$ $\mathrm{s}^{-1}$. In contrast, the anticyclonic eddies offshore start to move upstream at the same magnitude of speed. It is these opposite longshore movements of the cyclonic and anticyclonic eddies that tear themselves apart after the eddies elongate in the cross-shore direction. As is shown in Figure 5, the movement of these eddies also plays an important role in the material exchange between the current water and outer ocean water. The mechanisms that produce such eddy movement will be further studied in the next section.

Figure 6 shows the time changes of the basin-averaged values of the mean (zonal-averaged) kinetic energy (MKE), the eddy kinetic energy (EKE), and the available potential energy

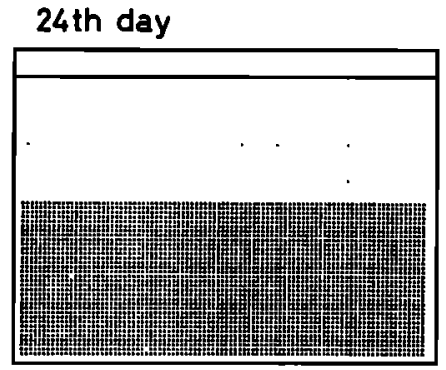

44 th day

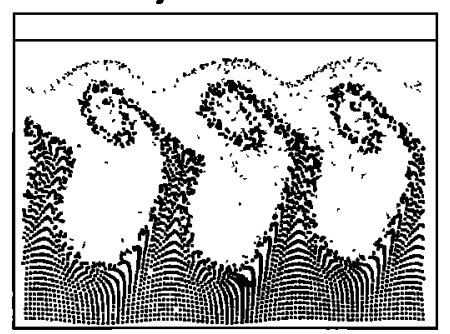

40 th day

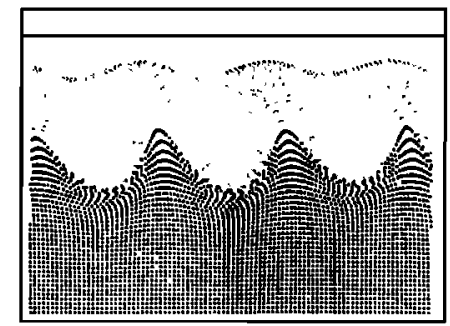

46 th day

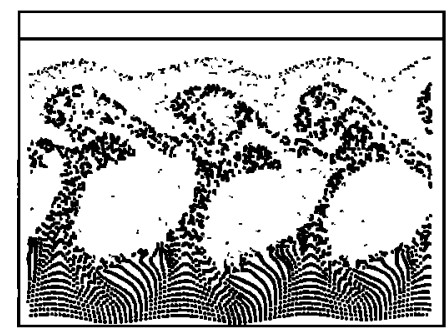

42nd day

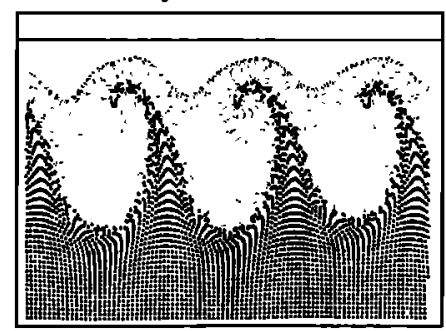

48 th day

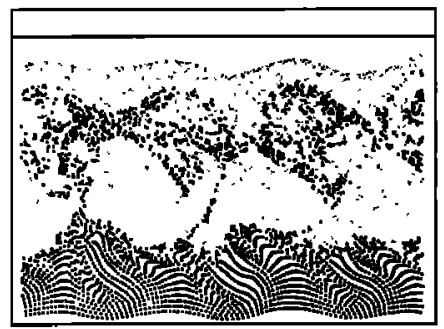

Fig. 5. Sequential patterns of passive particle movements in the surface level. Tracking started on day 24 before the onset of current's instability. Only the particles appearing inside the dotted window of Figure 4 (compare the 175-m panel for day 34) are shown in this figure. Light and dark marks represent particles initially arranged inside and outside the density current, respectively. 
(a)

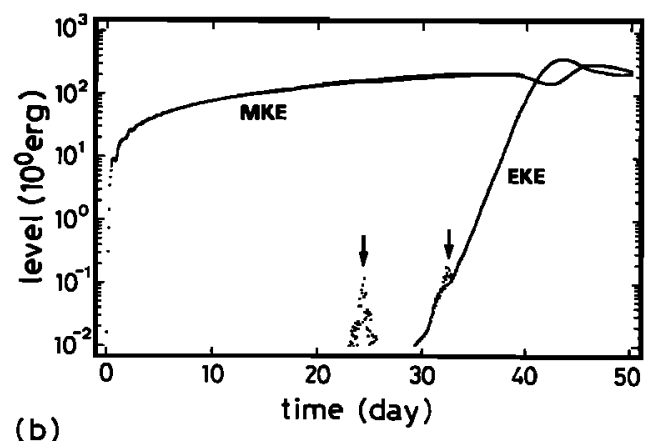

(b)

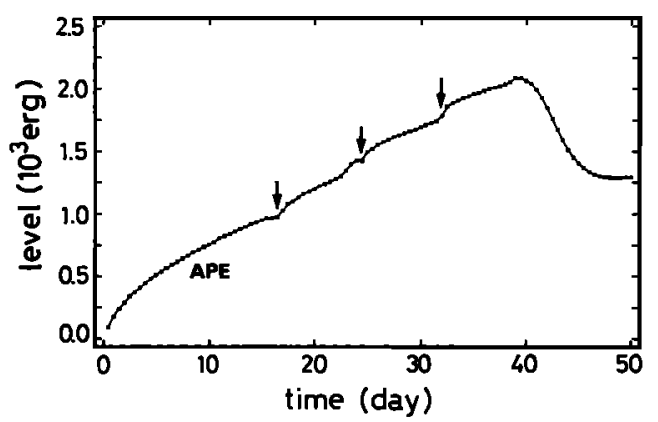

Fig. 6. (a) Time change of the zonal-averaged kinetic energy (MKE) and the eddy kinetic energy (EKE). Arrows indicate the occurrence of salinity reversal near the coast (see text for detailed explanation). (b) Time change of the available potential energy (APE) in the model basin. Arrows correspond to those shown in Figure $6 a$.

(APE). Figure 6a shows that the MKE accumulates steadily in the model basin after a few oscillations with an inertial period during the beginning of buoyancy supply. The kinetic energy of the disturbances (EKE), as was already noted in Figure 3, becomes significant from day 30 , and thereafter it increases exponentially with time until day 40 . An exponential time increase in the EKE implies that the unstable waves at this stage have small amplitudes and that the linear instability theory [e.g., Pedlosky, 1979] is applicable. Now supposing that

$$
\begin{aligned}
& u^{\prime}=\operatorname{Re}\left[U^{\prime} \exp i k(x-c t)\right] \\
& v^{\prime}=\operatorname{Re}\left[V^{\prime} \exp i k(x-c t)\right]
\end{aligned}
$$

where $k$ is the longshore wave number, $U^{\prime}$ and $V^{\prime}$ are the amplitudes of the deviated velocity components and $c=c_{r}$ $+i c_{t}$ is the complex phase speed, the linear growth rate of the unstable waves can be estimated by

$$
k c_{i}=\left(\ln E_{2}{ }^{\prime}-\ln E_{1}{ }^{\prime}\right) / 2\left(t_{2}-t_{1}\right)
$$

In (22), $E_{m}{ }^{\prime}=\left(U_{m}{ }^{2}+V_{m}{ }^{\prime 2}\right) / 2$ is the EKE values at time $t_{m}$ $(m=1,2)$. Using this estimation, we find that the linear growth rate of the unstable waves in Figure $6 a$ is 0.484 day $^{-1}$, i.e., an $e$-folding time of 2.1 days. The growth of the EKE slows down when the energy level of the EKE becomes comparable with that of the MKE around day 40 . After the day 43, the EKE even shows a tendency to decrease.

The growth of the density current is also accompanied by an increase in the APE as shown in Figure $6 b$. A remarkable decrease occurs in the APE around day 40 , at the same time as the EKE and MKE become comparable. In Figure $6 b$, it is also noteworthy that abrupt changes in the APE, as indicated by arrows, emerge periodically before the APE reaches its maximum value. It is of interest to clarify the background for these APE changes because any abrupt changes in the density field may break down the quasi-steady state of the density current and trigger grid-scale disturbances in the model's longshore direction. In fact, a comparison between Figure $6 a$ and Figure $6 \boldsymbol{b}$ shows that the abrupt changes in APE do raise the level of the EKE. Taking the case that occurred on day 32 as an example, we next investigate the cause of the abrupt APE changes.

In Figure 7, we show the detailed time changes of $\bar{S}, \bar{v}, \bar{w}$, and $\bar{u}$ obtained at three different depths $(12.5 \mathrm{~m}, 62.5 \mathrm{~m}$, and $125 \mathrm{~m}$ ) near the coast. Despite the fact that fresh water is supplied at a constant rate, a sudden reversal in the time change of $\bar{S}\left(\bar{S}_{t}>0\right)$ occurs at upper levels (Figure $7 a$ ). This salinity reversal continues only for about 2 days, and then the accumulation of fresh water resumes near the coast. At the lower level, the water is freshened during the reversal period. Time changes in $\bar{S}$ near the upper coast are determined by the following zonal-a veraged salinity budget equation:

$$
\bar{S}_{t}=(-B F)-\bar{v} \bar{S}_{y}-\bar{w} \bar{S}_{z}+\text { diffusive effect }
$$

where $B F$ indicates the fresh water supply and is given by (9), and $\bar{v} \bar{S}_{y}$ and $\bar{w} \bar{S}_{z}$ are the salinity fluxes through the horizontal and vertical surfaces of the upper coast region. The diffusive effect of the salinity can be neglected if $E_{h}$ is small such as in this case. Because of fresh water supply, $\bar{v}>0, \bar{w}>0, \overline{S_{y}}>0$, and $\bar{S}_{z}<0$ are valid at the upper coast region. Thus only the vertical advection term in (23) acts to increase the salinity (a) $\overline{\mathrm{S}}(\%)$

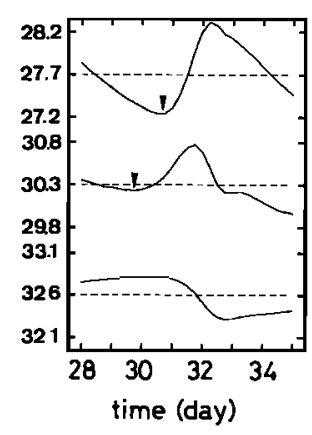

(c) $\overline{\mathrm{W}}\left(10^{-2} \mathrm{~cm} \cdot \mathrm{s}^{-1}\right)$

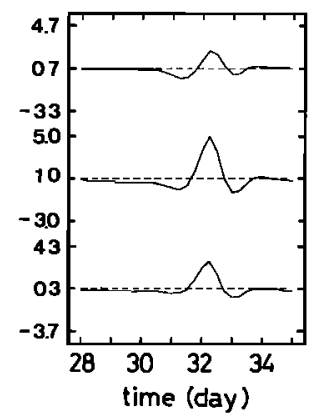

(b) $\bar{v}\left(\mathrm{~cm} \mathrm{~s}^{-1}\right)$

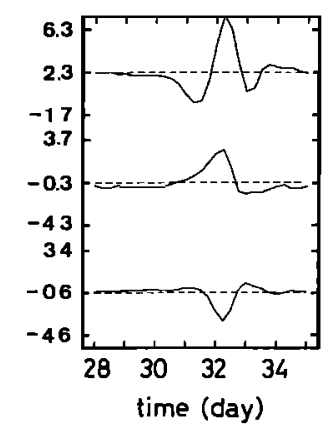

(d) $\bar{u}\left(\mathrm{~cm} \cdot \mathrm{s}^{-1}\right)$

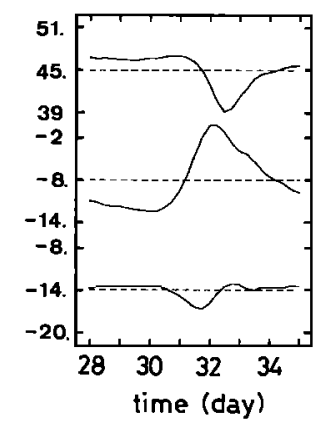

Fig. 7. Time changes in $(a) \bar{S},(b) \bar{v},(c) \bar{w}$, and $(d) \bar{u}$ in a typical salinity reversal duration. Values of the top, middle, and bottom curves are obtained at depths of $12.5 \mathrm{~m}, 62.5 \mathrm{~m}$ and $125 \mathrm{~m}$, respectively, near the coast $(y=4 \mathrm{~km})$. The arrows in Figure $7 a$ indicate that the salinity reversal in the middle level precedes that in the upper level. 
there. Dynamically, this implies that the penetration of the saltier water from lower levels is the only factor to cause $\bar{S}_{t}>0$. Excessive accumulation of fresh water near the upper coast tends to steepen the vertical gradient of $\bar{S}$, thus causing an increase in $\left|\bar{w} \overline{S_{z}}\right|$. Salinity reversal $\left(\bar{S}_{t}>0\right)$ occurs when $\left|\bar{w} \bar{S}_{z}\right|$ surpasses the other terms on the right-hand side of (23). As salinity continues to increase at the upper levels, $\left|\bar{w} \bar{S}_{z}\right|$ weakens and will eventually lose its dominance in (23). $\bar{S}_{1}<0$ then resumes. The penetration of saltier water from lower levels can also be comfirmed from the fact that the salinity reversal at a depth of $62.5 \mathrm{~m}$ precedes that at a depth of $12.5 \mathrm{~m}$ (compare the arrows in Figure $7 a$ ).

The salinity reversal forces significant adjustment in the velocity fields. The salinity increases near the upper coast causes the pressure gradient $\left(-\bar{P}_{y}\right)$ there to weaken. In the beginning, this decreases $\bar{v}$, and $\bar{w}$ through mass conservation, as can be seen in Figures $7 b$ and $7 c$ from day 30.5 to day 31.5. As $\left(-\bar{P}_{y}\right)$ continues to weaken, a strong decrease in $\bar{u}$ occurs around the day 31.5 (Figure $7 d$ ) due to a breakdown in the geostrophic balance of the density current. Once $\bar{u}$ decreases, the Coriolis force toward the coast weakens, and this causes the crossshore velocity $\bar{v}$ (and $\bar{w}$ ) to accelerate (day $31.5-32.2$ ). It is these sudden responses in the velocity field that promote the development of grid-scale disturbances (originating from numerical errors) in the model basin. After $\bar{S}_{t}<0$ resumes on day 32.2 , the velocity field settles down again to a new quasisteady state.

In cases where $E_{h}$ is large, that is, either when the freshwater supply is small or the diffusive effect in (23) is large, no abrupt changes of APE appear in the numerical experiments. This is simply because the excessive accumulation of fresh water is not possible near the upper coast. Moreover, our numerical experiments reveal that when $E_{h}$ exceeds a critical value (see section 5), sudden changes in the salinity and velocity fields will inevitably occur through the above processes, triggering grid-scale disturbances in the longshore direction. Depending on the vertical shear strength of the basic mean current, the grid-scale disturbances thus triggered may be suppressed by the dissipative effect, such as the case on day 24 in Figure $6 a$, or they may eventually evolve into large-scale unstable waves as on day 32. The conditions determining the development of the grid-scale disturbances will be studied in section 5 .

\section{Energetics of THE UnStable Waves}

In order to understand the dynamics of unstable density currents, it is important to clarify the energetics involved in the instability process. In this section we discuss the energy transfers among the MKE, EKE, and potential energy (PE) of the density current. The energy equation for MKE can be derived from multiplying (1) by $\bar{u}$ and (2) by $\bar{v}$ and then adding them together. In the same way, multiplying (1) by $u^{\prime}$ and (2) by $v^{\prime}$ and adding gives the energy equation for the EKE. Multiplying (5) by $\beta g z$ gives the energy equation for the PE. The results are as follows:

$$
\begin{gathered}
K e_{t}=\langle K m, K e\rangle+\langle P e, K e\rangle+\text { dissipation of EKE } \\
K m_{t}=-\langle K m, K e\rangle+\langle P m, K m\rangle+\text { dissipation of MK } \\
P_{t}=-\langle P, K\rangle+(B S)+\text { dissipation of } P E
\end{gathered}
$$

where

$$
K m=\iint\left(\bar{u}^{2}+\bar{v}^{2}\right) d y d z / 2 A_{0}
$$

$$
\begin{gathered}
K e=\iint\left(\overline{u^{\prime 2}}+\overline{v^{\prime 2}}\right) d y d z / 2 A_{0} \\
P=\iint(\bar{\rho} g z) d y d z / A_{0} \\
\langle K m, K e\rangle=-\iint\left(\overline{u^{\prime} v^{\prime}} \bar{u}_{y}+\overline{u^{\prime} w^{\prime}} \bar{u}_{z}+\overline{v^{\prime} v^{\prime}} \bar{v}_{y}\right. \\
\left.+\overline{v^{\prime} w^{\prime}} \bar{v}_{z}\right) d y d z / A_{0} \\
\langle P e, K e\rangle=-\iint\left(g \overline{\rho^{\prime} w^{\prime}}\right) d y d z / A_{0} \\
\langle P m, K m\rangle=-\iint(g \bar{\rho} \bar{w}) d y d z / A_{0} \\
\langle P, K\rangle=\langle P e, K e\rangle+\langle P m, K m\rangle
\end{gathered}
$$

In the above equations, $K m, K e$, and $P$ are the basin-averaged values of MKE, EKE, and PE, respectively. $A_{0}$ is the area of the vertical cross-shore section. $\langle K m, K e\rangle$ represents a conversion of MKE to EKE through Reynolds stresses and is the energy source for barotropic instabilities. $\langle P e, K e\rangle$ represents a conversion from the eddy potential energy to EKE, which causes baroclinic instabilities. $\langle P m, K m\rangle$ is a conversion from the mean potential energy to the MKE, and $(B S)$ is the potential energy supply from the buoyancy influx along the upper coast. It becomes evident by adding (24), (25), and (26) that

$$
(K e+K m+P)_{t}=(B S)+\text { dissipation of MKE + EKE + PE }
$$

That is, the time change of the total energy in the basin is determined by the sum of the potential energy supply and the dissipation of the total energy.

Figure 8 shows the time changes of the energy balances of (24), (25), and (26) for the case discussed in the preceding section. We next investigate these balances by separating them into three different stages according to the different underlying dynamics.

Energy accumulation stage (day 0 to day 33). The buoyancy-driven density current at this stage grows steadily with insignificantly small variation in the longshore direction. As is shown in Figure $8 a$, the terms of (24) are negligible in the model basin. The noticeable feature at this stage is the sudden periodic changes in $P_{t}$ (compare arrows in Figure $8 c$ ) caused by the salinity reversal near the coast discussed in the last section. Since the buoyancy supply rate is held constant, these sudden changes directly effect $\langle P m, K m\rangle$, the energy transfer from the mean potential field to the mean kinetic field, and eventually cause temporal changes in the MKE field (compare arrows in Figure 8 b). As was discussed in the last section, each of these sudden changes promotes the development of the grid-scale disturbances.

Linear developing stage of the unstable waves (day 33 to day 40). At this stage, the triggered grid-scale disturbances become organized and evolve into large-scale unstable waves through the nonlinear cascading process. As is found in Figure $6 a$, the unstable waves at this stage have small amplitude, and their growth can be described by the linear instability theory. From Figure $8 a$ it is clear that $\langle P e, K e\rangle$, an energy transfer from the eddy potential field, is the source which feeds the growth of the EKE. In other words, the density current develops unstably through baroclinic instability. This finding is 
(a)
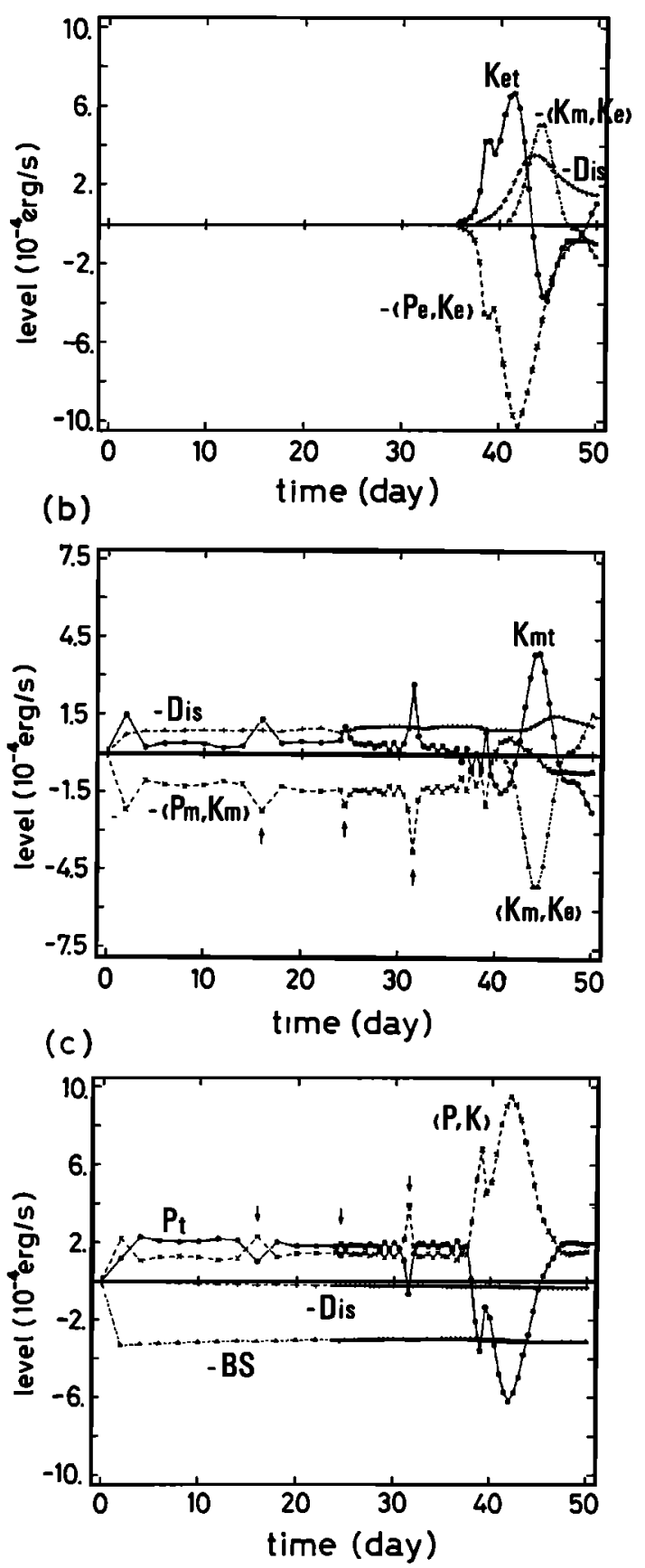

Fig. 8. Time changes in energy balance equations for $(a)$ EKE, (b) MKE, and (c) PE Terms in the figures are defined in (24)-(27) in the text. Arrows in Figures $8 b$ and $8 c$ indicate the occurrence of salinity reversals.

consistent with the result in Figure 4 where baroclinic instability was confirmed from the fact that the vertical phase of $v^{\prime}$ patterns leans against the vertical shear of the mean current.

Nonlinear stage of the unstable waves (after day 40). The energy level of the EKE at this stage reaches the same order as that of the MKE (compare Figure $6 a$ ), and the unstable waves attain such a large amplitude that nonlinear effects can no longer be neglected. In Figure $8 a$ a negative $\langle K m, K e\rangle$ is found from day 40 to day 47 , indicating that an energy transfer from the EKE to the MKE takes place. Contrary to the role of positive $\langle K m, K e\rangle$, which reinforces the eddy field through barotropic instabilities of the mean flow, a negative $\langle K m, K e\rangle$ suggests an energy feedback from the eddy field to the mean flow due to Reynolds stresses. For this reason the negative $\langle K m, K e\rangle$ is sometimes referred to as "negative viscosity" [e.g., Orlanski and Cox, 1973].

It is of interest here to investigate what kind of mean flows are induced by the negative $\langle K m, K e\rangle$ in the model basin. From the definition of $(27 d)$,

$$
\begin{aligned}
\langle K m, K e\rangle=-\iint\left(\overline{u^{\prime} v^{\prime}} \bar{u}_{y}+\overline{u^{\prime} w^{\prime} \bar{u}_{z}}\right. & +\overline{v^{\prime} v^{\prime}} \bar{v}_{y} \\
& \left.+\overline{v^{\prime} w^{\prime}} \bar{v}_{z}\right) d y d z / A_{0}
\end{aligned}
$$

In the density current system under consideration, the numerical result shows that the first term dominates the other terms on the right-hand side of (29). Thus $\langle K m, K e\rangle$ can be estimated from the product of the horizontal shear of the mean current $\bar{u}_{y}$ and the zonal-averaged Reynolds stress, $\overline{u^{\prime} v^{\prime}}$. Figure $9 a$ shows the time change of $-\int_{-H_{0}}^{0}\left(\overline{u^{\prime} v^{\prime}} \bar{u}_{y}\right) d z / H_{0}$ as a function of offshore distance. After the unstable waves develop into the nonlinear stage, a large negative value appears in the region centered $50 \mathrm{~km}$ offshore where the unstable waves have their maximum amplitude as shown in the day 40 pattern of Figure 4. Thus we find that negative viscosity is mainly produced where the waves are most unstable. Around day 45 a new negative value region emerges further offshore. This negative value is induced by the energy transfer from the eddies which split offshore at the nonlinear stage (compare the day 46 pattern in Figure 4).

The zonal-averaged Reynolds stress, $\overline{u^{\prime} v^{\prime}}$, is also closely related to the acceleration of the mean current. After averaging the momentum equation (1) in the longshore vertical section and neglecting its small dissipative effect, we have

$$
\begin{aligned}
\frac{1}{H_{0}} \int_{-H_{0}}^{0} \bar{u}_{z} d z=-\frac{1}{H_{0}} \int_{-H_{0}}^{0}\left(\overline{u^{\prime} v^{\prime}}\right)_{y} d z & -\frac{1}{H_{0}} \int_{-H_{0}}^{0}(\bar{u} \bar{v})_{y} d z \\
& +\frac{1}{H_{0}} \int_{-H_{0}}^{0} f \bar{v} d z
\end{aligned}
$$

As in (29), the first term on the right-hand side of (30) dominates the other terms. That is, the acceleration of the mean current $\int_{-H_{0}}^{0} \bar{u} d z / H_{0}$ is approximately determined by the $y$ derivative of the zonal-averaged Reynolds stress. The distribution of $-\int_{-H_{0}}^{o}\left(\overline{u^{\prime} v^{\prime}}\right)_{y} d z / H_{0}$ is shown in Figure $9 b$ in the same fashion as Figure $9 a$. At the nonlinear stage, a positive value appears within the coastal region extending $50 \mathrm{~km}$ offshore. Since the basic density current in this region moves in the $+x$ direction, this positive value suggests that the vertically averaged mean current near the coast is further reinforced. In contrast, a negative value of $-\int_{-H_{0}}^{0}\left(\overline{u^{\prime} v^{\prime}}\right)_{y} d z / H_{0}$ is found in the offshore region beyond $50 \mathrm{~km}$. This suggests that a mean current moving in the $-x$ direction is induced on the offshore side of the basic current. These two facts are easily verified in Figure 10, which shows the time changes of $\bar{u}$ in the cross-shore section. Figure 10 shows that before the onset of instability the density current is strong and confined near the upper coast with a compensating countercurrent flowing underneath. As the unstable waves develop into the nonlinear stage, we find that the positive value of $-\int_{-H_{0}}^{0}\left(\overline{u^{\prime} v^{\prime}}\right)_{y} d z / H_{0}$ turns the whole depth of the current near the coast to the $+x$ direction, while offshore, a mean flow moving in the opposite direction is induced by the negative value of $-\int_{-H_{0}}^{0}\left(\overline{u^{\prime} v^{\prime}}\right)_{y}$ $d z / H_{0}$. 
(a)

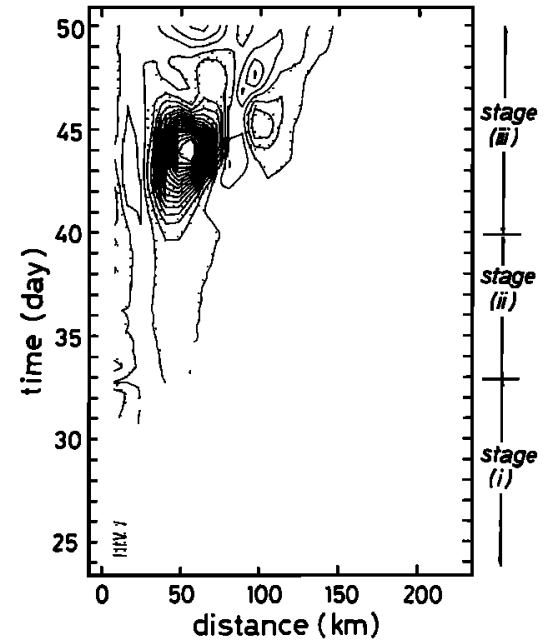

(b)

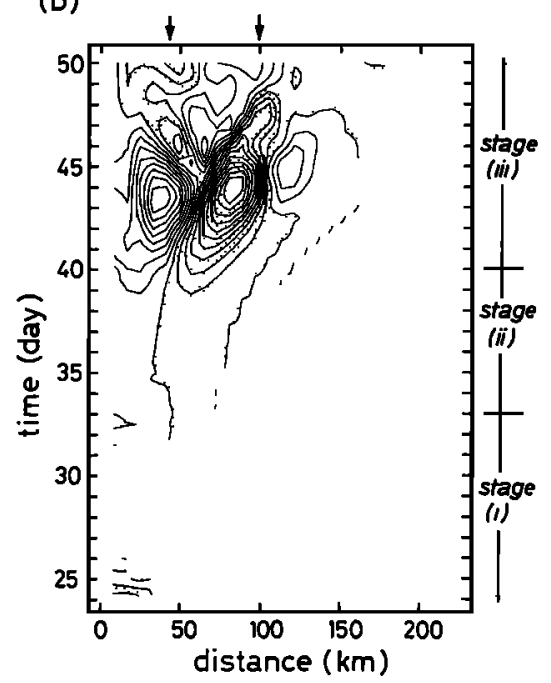

Fig. 9. (a) Time changes of $-\int_{-H_{0}}^{0}\left(\overline{u^{\prime} v^{\prime}} \bar{u}_{y}\right) d z / H_{0}$ as a function of the cross-shore distance. (b) Time changes of $-\int_{-H_{0}}^{0}$ $\left(\overline{u^{\prime} v^{\prime}}\right)_{y} d z / H_{0}$ as a function of the cross-shore distance. In the figures, $x$ axes show the offshore distance from the coast, and the shaded regions indicate negative values. Contour value intervals are $6 \times 10^{-3} \mathrm{~cm}^{2} \mathrm{~s}^{-3}$ and $1.4 \times 10^{-5} \mathrm{~cm} \mathrm{~s}^{-2}$ in Figures $9 a$ and $9 b$, respectively.

In Figure 5 we mentioned that after the unstable waves evolve to their nonlinear stage, the cyclonic eddies near the coast and the anticyclonic eddies offshore begin to move in the $+x$ and $-x$ direction, respectively. These eddies' movements can also be seen well in Figures $11 a$ and $11 b$, in which the time changes of the zonal distribution of $v^{\prime} 45 \mathrm{~km}$ offshore (the center of the cyclonic eddies) and $100 \mathrm{~km}$ offshore (the center of the anticyclonic eddies), are shown, respectively. Although the unstable waves are nearly stationary at the linearly developing stage, we notice that the fully developed cyclonic eddies near the coast start to move in the $+x$ direction and the anticyclonic eddies offshore start to move in the $-x$ direction after day 43. Both of the eddies have a speed of $10 \mathrm{~cm} \mathrm{~s}^{-1}$ and move in the same directions as the induced mean flows (Figure 10). These suggest that the eddies' movements are possibly caused by the advection of the mean flows.

As a result, we find that after the unstable waves evolve into the nonlinear stage, the energy transfers through the "negative viscosity" will induce mean flows moving in the opposite direction at the edge of the density current. The advections of these mean flows will cause the fully developed unstable waves to detach in the cross-shore direction, resulting in water mass exchanges across the density current.

To conclude this section, we show the averaged energy transfer flows during the three stages in Figure 12. The open arrows in Figure 12 indicate transfers between different types of energy, and the solid arrows indicate energy dissipation. The time change value is written under the name of each energy. The differences in the dynamics at each stage described above are concisely summarized in this figure.

\section{The $E_{h}$ Dependence of Density Currents}

In sections 3 and 4 we studied the characteristics of an unstable density current in a representative case. As we found in section 3, the occurrence of the initial grid-scale disturbances in the longshore direction are sensitive to the velocity and salinity structures in the cross-shore section. Scaling analysis of the $x$ independent governing equations in section 2

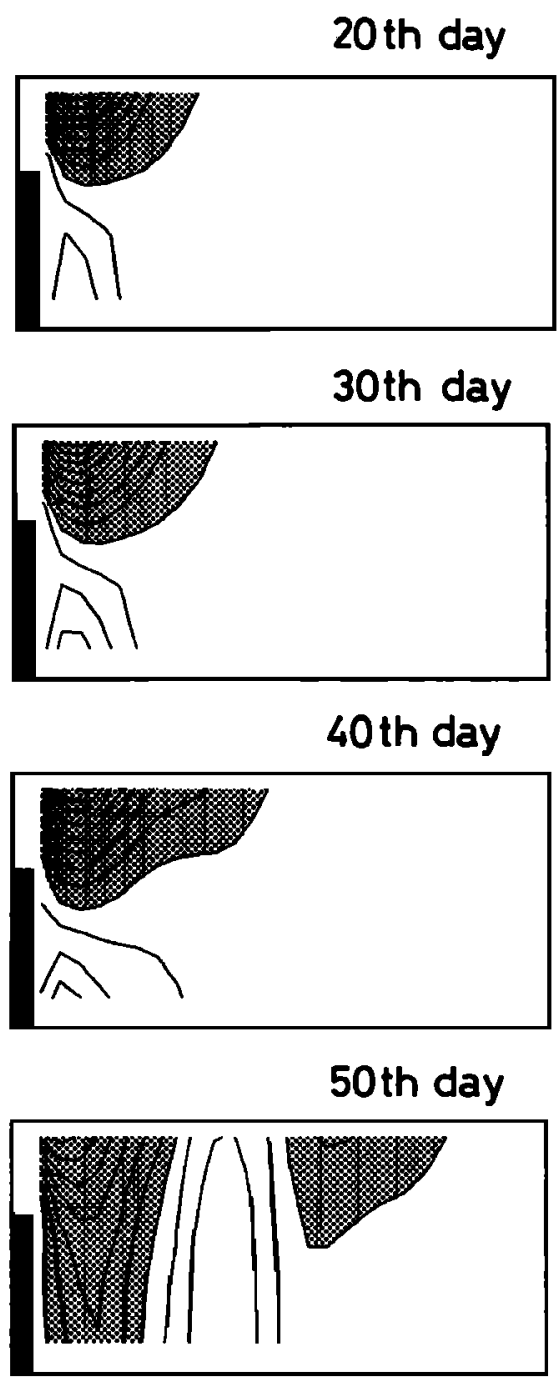

Fig. 10. Sequential patterns of zonal-averaged current $\bar{u}$ in the cross-shore vertical section. Flows in the shaded regions move with the coast to the right. Contour value interval is $10 \mathrm{~cm} \mathrm{~s}^{-1}$. 
(a)

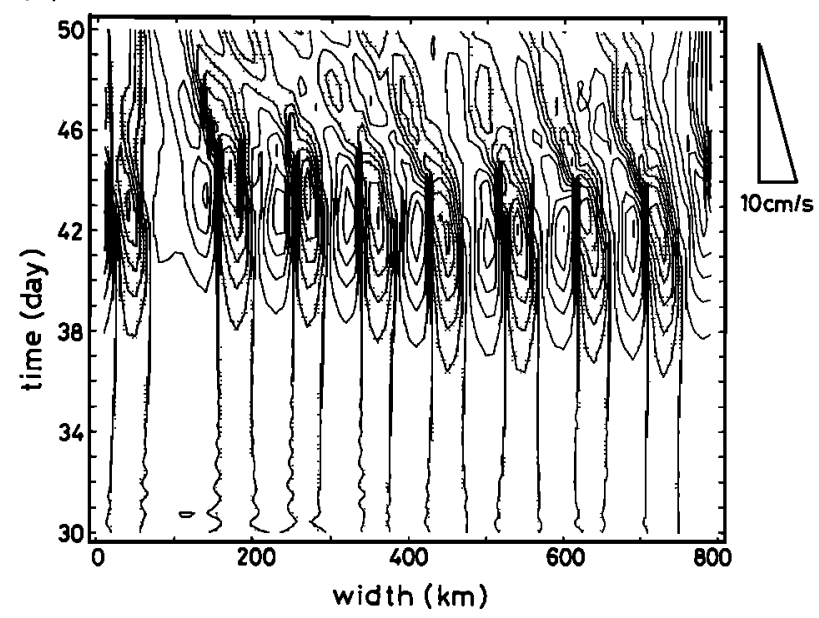

(b)

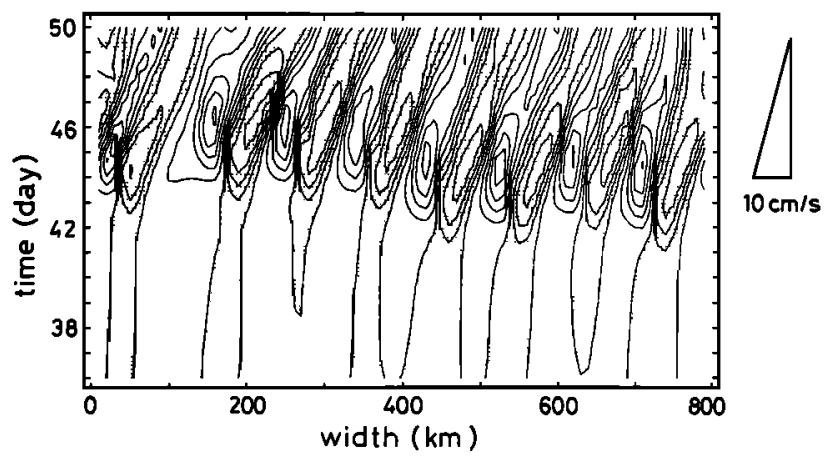

Fig. 11. Time changes of zonal distribution of $v^{\prime}$ at offshore sites (a) $45 \mathrm{~km}$ and $(b) 100 \mathrm{~km}$ offshore. (See arrows in Figure $9 b$ for locations.) The contour interval is $10 \mathrm{~cm} \mathrm{~s}^{-1}$ and the shaded areas indicate positive values. The triangles on the right-hand side give the typical phase speed of the unstable waves.

suggested that the horizontal Ekman number $E_{h}=A_{h} f /$ $\left(g^{\prime} Q_{e}\right)^{2 / 3}$ is the crucial external parameter in determining the cross-shore structures. In this section, we examine how the external parameter $E_{h}$ influences the stability of the buoyancydriven density currents in the numerical experiments. The $E_{h}$ dependence of the currents' instability is then compared with the results from a frictional two-layer quasi-geostrophic model.

In order to examine the $E_{h}$ dependence, we performed more numerical experiments using different combinations of the horizontal eddy viscosity $A_{h}$ and the discharge rate $Q_{e}$ given in Table 1. Figure 13 shows the stability of all the experimental cases on the $A_{h}-Q_{e}$ plane. In Figure 13, a solid symbol indicates an unstable case in which the EKE grows exponentially with time, while an open symbol indicates a stable case in which no EKE emerges in the basin. Dashed lines satisfy $Q_{e} \propto A_{h}{ }^{3 / 2}$ and thus are the isopleths of $E_{h}$. The result in Figure 13 suggests that it is reasonable to separate the stable and unstable regions on the $A_{h}-Q_{e}$ plane by a critical $E_{h}$ isopleth between the two broken lines, that is, $0.354<E_{h c}<$ 0.566 . This critical isopleth is shown conceptually by a solid line in Figure 13. The existence of the critical $E_{h}$ value is consistent with the scaling analysis result in section 2 that $E_{h}$ is the crucial external parameter to determine the stability of the buoyancy-driven density currents.

In the representative case discussed in the previous sections,

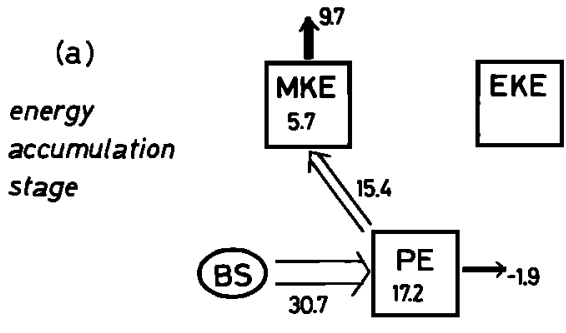

(b)

linear
developing
stage

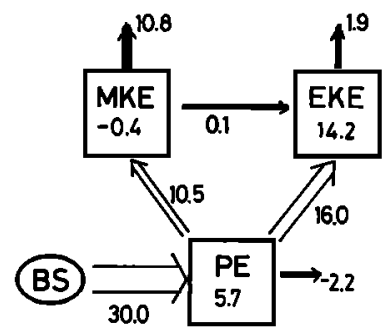

(c)

nonlinear
stage

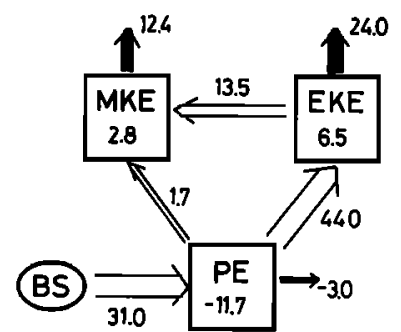

Fig. 12. Energy flow diagrams showing energy transfers averaged at three stages of the unstable density current: $(a)$ energy accumulation stage, (b) linear developing stage of the unstable waves, and $(c)$ nonlinear stage of the unstable waves. Time change values are shown under the name of each energy. The values are in units of $10^{-s}$ ergs $\mathrm{s}^{-1}$.

the unstable waves on the density current had a wave number $n=8$ (compare Figure 4). In the numerical experiments, the wave numbers $n$ of the unstable wave change as $E_{h}$ varies. Some examples of the unstable waves with different wave

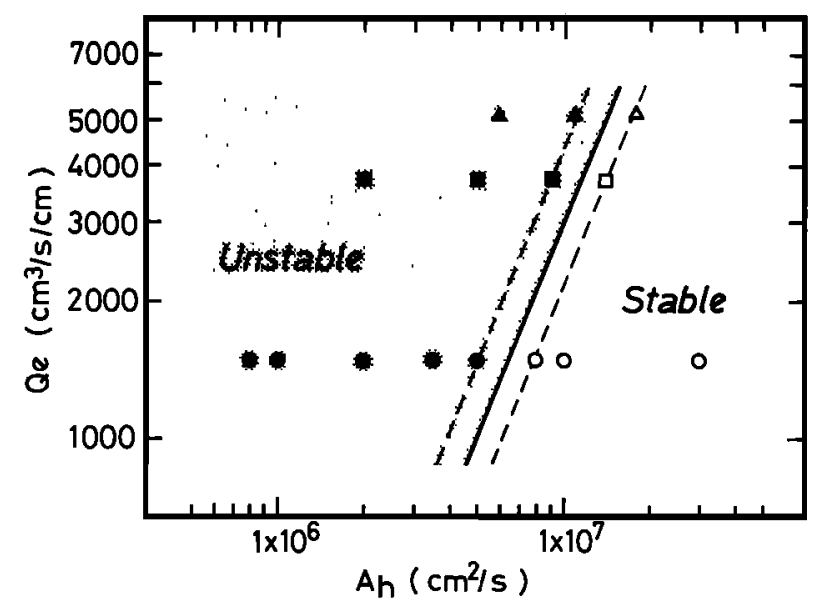

Fig. 13. Stability of the density currents as a function of external parameters $A_{h}$ and $Q_{c}$. Open symbols indicate stable cases found in the numerical experiments, while solid ones indicate unstable cases. Straight lines are the isopleths of the horizontal Ekman number $E_{h}=$ $A_{h} f /\left(g^{\prime} Q_{e}\right)^{2 / 3}$. The density currents grow unstably in the shaded region. 
numbers are shown in Figure 14. A variation in $E_{h}$ also affects the linear growth rate of the unstable waves. In Figure $15 a$ we summarize the $E_{h}$ dependence of the wave number and the linear growth rate for all the unstable cases obtained in the numerical experiments. From Figure $15 a$, we notice that the wave number decreases in general as $E_{h}$ becomes larger and that the linear growth rate also decreases with increasing $E_{h}$. The linear growth rate becomes zero (stable case) as $E_{h}$ exceeds the critical value $E_{h c}$, which corresponds to the solid line in Figure 13.

As was mentioned in the previous sections, the unstable waves at the linear developing stage have small amplitudes, and their development follows the linear instability theory. To further investigate the role of $E_{h}$ in determining the wave number and the growth rate of the unstable waves, it is of interest as a comparison to consider the density current in a two-layer quasi-geostrophic model including the horizontal diffusive effect. Since the unstable waves grow via baroclinic instability at their linear developing stage (section 4), this implies that the vertical shear of the density current is more essential than the current's horizontal shear in determining the wave number and the growth rate of the unstable waves. Thus we neglect the current's horizontal shear in the following analysis.

Suppose that $U_{j}$ and $\rho_{j}$ are the velocity and density of the basic current in the $j$ th layer $(j=1,2)$. The small-amplitude perturbations on the basic current must then satisfy the following nondimensional potential vorticity equation,

$$
\left(\frac{\partial}{\partial t}+U_{j} \frac{\partial}{\partial x}\right) q_{j}-F_{j}(-1)^{j} U s \frac{\partial \phi_{j}}{\partial x}=\left(\frac{E_{h}}{\varepsilon}\right)\left(\frac{\partial^{4}}{\partial x^{4}}+\frac{\partial^{4}}{\partial y^{4}}\right) \phi_{j}
$$

(a)

$$
n=6
$$

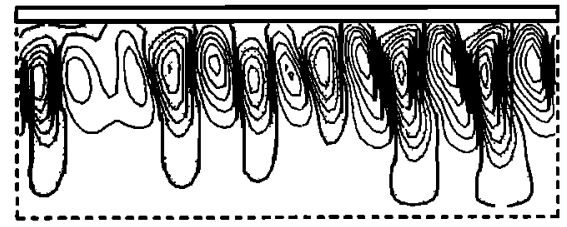

(b)

$$
n=5
$$

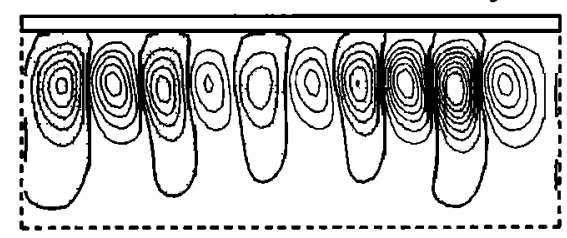

(c)

$$
n=4
$$

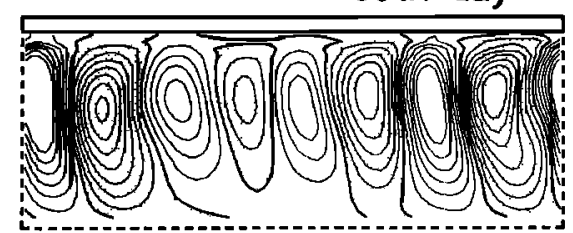

Fig. 14. Examples of the unstable waves with different wave numbers (patterns shown in the same way as in Figure 4): (a) $n=6$, where $E_{h}=0.077\left(Q_{e}=3750 \mathrm{~cm}^{2} \mathrm{~s}^{-1}\right.$ and $\left.A_{h}=2 \times 10^{6} \mathrm{~cm}^{2} \mathrm{~s}^{-1}\right) ;(b) n=5$, where $E_{h}=0.355\left(Q_{e}=1500 \mathrm{~cm}^{2} \mathrm{~s}^{-1}\right.$ and $\left.A_{h}=5 \times 10^{6} \mathrm{~cm}^{2} \mathrm{~s}^{-1}\right)$; and $(c) n=4$, where $E_{h}=0.338\left(Q_{e}=5250 \mathrm{~cm}^{2} \mathrm{~s}^{-1}\right.$ and $A_{h}=11$ $\left.\times 10^{6} \mathrm{~cm}^{2} \mathrm{~s}^{-1}\right)$. (a)

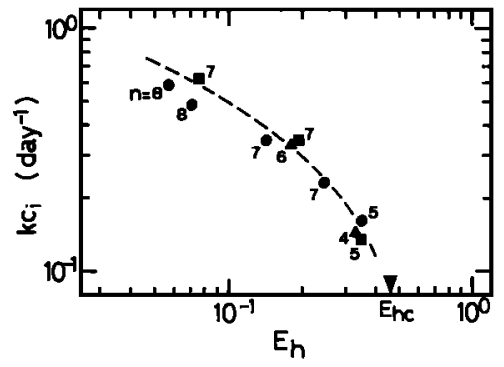

(b)

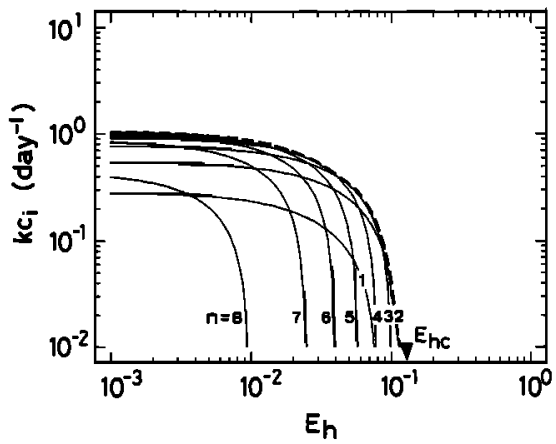

Fig. 15. The linear growth rates $\left(k c_{i}\right)$ and the wave numbers $n$ of the unstable waves as a function of $E_{h}$. $(a)$ Numerical results. The number attached to the symbols indicates the wave number, and $E_{h c}$ indicates the numerically obtained critical $E_{h}$ value. $(b)$ Results in a frictional two-layer quasi-geostrophic model. In Figure $15 b$, $n \equiv k W / 2 \pi R o$ has the same definition as in Figure $15 a$. The dashed line gives the maximum growth rate and the wave number of the most unstable waves. The external parameters for Figure $15 b$ are $\alpha=0.4$, $\varepsilon=0.1, m=0.38$ and $U s=2.0$ (in dimensional unit $0.005 \mathrm{~s}^{-1}$ ).

where

$$
\begin{gathered}
q_{j}=\left(\frac{\partial^{2}}{\partial x^{2}}+\frac{\partial^{2}}{\partial y^{2}}\right) \phi_{j}-(-1)^{j} F_{j}\left(\phi_{2}-\phi_{1}\right) \quad U S=U_{2}-U_{1} \\
F_{1}=f^{2} L^{2} / g^{\prime} H_{0} \alpha \quad F_{2}=f^{2} L^{2} / g^{\prime} H_{0}(1-\alpha)
\end{gathered}
$$

In $(31), \phi_{j}$ is the stream function of the perturbances, $\alpha$ is the ratio of the depth of the upper layer to the entire depth, $\varepsilon=U / f L$ is the Rossby number, and $E_{h}=A_{h} / f L^{2}$ is the horizontal Ekman number, where $L$ and $U$ are the length and velocity scales of the basic current, respectively. Note that (31) reduces to the Phillips' two-layer model [Pedlosky, 1979] when $E_{h}=0$.

Assuming the normal mode solution, $\phi_{j}=A_{j} \cos (m y)$ exp [ik(x-ct)], with $c, k$, and $m$ as the complex phase speed and wave numbers in $x$ and $y$ directions, (31) becomes

$$
\begin{aligned}
{\left[\left(c-U_{1}\right)\left(K^{2}+F_{1}\right)+F_{1} U s+i E_{h} K^{4} / \varepsilon k\right] } & A_{1} \\
& -F_{1}\left(c-U_{1}\right) A_{2}=0
\end{aligned}
$$

$\left[\left(c-U_{2}\right)\left(K^{2}+F_{2}\right)-F_{2} U s+i E_{h} K^{4} / \varepsilon k\right] A_{2}$

$$
-F_{2}\left(c-U_{2}\right) A_{1}=0
$$




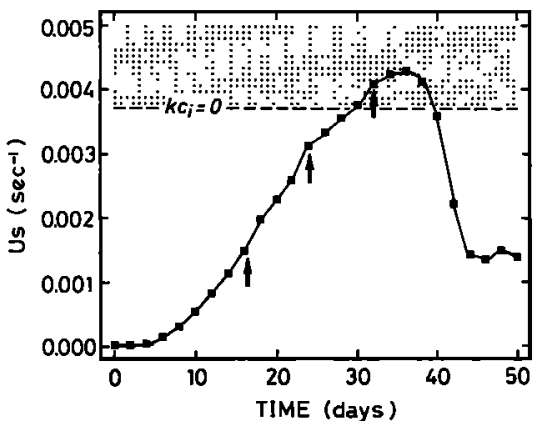

Fig. 16. Time changes of the mean vertical shear for the density current obtained in the representative case. Arrows indicate the occurrence of the salinity reversal near the coast (compare Figure 6). The dashed line at $U s=0.0037 \mathrm{~s}^{-1}$ gives the threshold value for the basic current, which is obtained from (34) when the external parameters are set as $E_{h}=0.071, \alpha=0.4, \varepsilon=0.4, n=8$ and $m=0.38$. The basic current evolves unstably only when $U s$ exceeds the threshold value (shaded area).

where $K^{2}=k^{2}+m^{2}$. Nontrivial solutions for $A_{j}$ requires

$$
\begin{array}{r}
c=U_{2}+\frac{U s\left(K^{2}+2 F_{2}\right)}{2\left(K^{2}+F_{1}+F_{2}\right)}-\frac{i E_{h} K^{2}\left(2 K^{2}+F_{1}+F_{2}\right)}{\varepsilon k 2\left(K^{2}+F_{1}+F_{2}\right)} \\
\pm \frac{1}{2\left(K^{2}+F_{1}+F_{2}\right)}\left[-\frac{E_{h}^{2} K^{4}}{\varepsilon^{2} k^{2}}\left(F_{1}+F_{2}\right)^{2}\right. \\
\left.+2 U s \frac{i E_{h}}{\varepsilon k} K^{4}\left(F_{1}-F_{2}\right)-U s^{2}\left(4 F_{1} F_{2}-K^{4}\right)\right]^{1 / 2}
\end{array}
$$

This dispersion relation gives the linear growth rate in the following formula,

$$
\begin{aligned}
& k c_{1}=-\frac{E_{h} K^{2}\left(2 K^{2}+F_{1}+F_{2}\right)}{2 \varepsilon\left(K^{2}+F_{1}+F_{2}\right)}+\frac{1}{2\left(K^{2}+F_{1}+F_{2}\right)} \\
& \cdot\left\{\frac{1}{2}\left[\left(a^{2}+b^{2}\right)^{1 / 2}-a\right]\right\}^{1 / 2}
\end{aligned}
$$

where

$$
\begin{gathered}
a=-E_{h}{ }^{2} K^{4}\left(F_{1}+F_{2}\right)^{2} / \varepsilon^{2}-U s^{2} k^{2}\left(4 F_{1} F_{2}-K^{4}\right) \\
h=2 k U s E_{h} K^{4}\left(F_{1}-F_{2}\right) / \varepsilon
\end{gathered}
$$

When $U s, k, m$, and $x$ are externally chosen, the linear growth rate $k c_{1}$ can be solved as a function of $E_{h}$ by (34). It is illuminating to consider first a special case in which $F_{1}=F_{2}$ (i.e., $\alpha=0.5$ ). From (34) it can be easily confirmed that

$$
E_{h}<\varepsilon k U s\left(2 F_{1}-k^{2}-m^{2}\right)^{1 / 2} / 2\left(k^{2}+m^{2}\right)^{3 / 2}
$$

must be satisfied for $k c_{1}>0$. That is, for a specified wave number $k$, instability will be suppressed by the diffusive effect when the horizontal Ekman number exceeds the value given by the right-hand side of (35). When the cross-shore perturbation scale $m^{-1}$ is given (e.g., by the width of the basic current), (35) shows that a critical value in $E_{h}$ exists at $k^{2}=2 F_{1}$ $-s^{2}$ :

$$
E_{h c}=\varepsilon U s\left[\left(2 F_{1}-s^{2}\right)\left(s^{2}-m^{2}\right)\right]^{1 / 2} / 2\left(2 F_{1}-s^{2}+m^{2}\right)^{3 / 2}
$$

where $s^{2}=\left(4 F_{1}^{2}-2 F_{1} m^{2}+m^{4}\right)^{1 / 2}$. Beyond this critical value, the current system is stable to perturbations of any longshore scales. It can also be shown from (35) that no unstable wave with $k^{2}>2 F_{1}-m^{2}$ can develop. This shortwave cutoff is the same as that deduced in the inviscid two-layer model [Pedlosky, 1979].
Figure $15 b$ gives a detailed result of (34), showing the dependency of $k c_{\imath}$ on $E_{h}$ for the different basin wave numbers $n$. Here, $n(=k W / 2 \pi L$, where $W$ is the basin width) corresponds to the wave number used in Figure 15a. A critical horizontal Ekman number $E_{h c}$ exists in Figure $15 b$, above which no waves of $k c_{i}>0$ appear in the current system. Figure $15 b$ also shows that the existence of the shortwave cutoff mentioned above has prohibited perturbations with $n>8$ from growing. Since unstable waves with the largest growth rates will actually emerge in the basin, the dashed line in Figure $15 b$ gives the actual growth rate and its corresponding wave number. It is clear that an increase in $E_{h}$ leads to a decrease in $n$ as well as in $k c_{i}$, which is qualitatively consistent with the numerical results of Figure 15a. Moreover, Figure $15 b$ suggests that although the perturbation with $n=1$ has a nonzero growth rate, it will not actually emerge in the current system because of its weak growth rate. This fact can qualitatively explain why long unstable waves with $n<4$ are not obtained in the numerical experiments (Figure 15a).

Another fact revealed by the quasi-geostrophic model is that even when the model's horizontal Ekman number $E_{h}$ is smaller than $E_{h c}$, the density current will not become unstable unless the vertical shear of its longshore velocity, $U s$, exceeds a certain value. For example, Figure 16 shows the evolution of $U s$ as a function of time for the numerical case discussed in the previous sections. Calculation of (34) based on the parameters corresponding to the current's values on day $30\left(E_{h}=\right.$ $0.071, \alpha=0.4, \varepsilon=0.4, n=8$, and $m=0.38$ ) shows that $U s=0.0037 \mathrm{~s}^{-1}$ is a threshold value for the instability, below which the density current is stable even in the presence of grid-scale disturbances. This result in turn explains why the grid-scale disturbances appearing on days 17 and 24 did not grow (compare the arrows in Figure 16), while the grid-scale disturbances on day 32 evolved into the large-scale unstable waves after the vertical shear of the basic current exceeded the threshold value.

Comparison between Figures $15 a$ and $15 b$ reveals that the general features of $E_{h}$ dependency of $k c_{l}$ and $n$ of the unstable density currents can be explained by the quasi-geostrophic instability theory despite its simplicity and constraints. However, it is important to mention that the quasi-geostrophic theory only provides information on the wave numbers and growth rates of the fastest-growing unstable waves. The nonlinear aspects of the unstable waves and their effect on material exchange across the density current as obtained in the numerical experiments can not be derived from the quasigeostrophic instability theory.

\section{Conclusions and Discussion}

Using the three-dimensional primitive equation model, we investigated the characteristics of density currents induced by buoyancy flux supply along the upper coast. Special attention was focused on the unstable cases in which grid-scale disturbances emerging on the density current develop into largescale unstable waves. The major conclusions from the present study are as follows.

Scaling analysis of the $x$-independent governing equations revealed that when the vertical eddy diffusivity is small, the horizontal Ekman number $E_{h}=A_{h} f /\left(g^{\prime} Q_{e}\right)^{2 / 3}$ is the crucial external parameter determining the density and velocity patterns in the cross-shore section. Since the stability of density currents depend on these cross-shore patterns, we also expected 
the $E_{h}$ would be a factor determining the currents' stability. This expectation was confirmed in the numerical experiments by using different combinations of $A_{h}$ and $Q_{e}$. It is found numerically that a critical $E_{h}$ value exists between 0.354 and 0.566 . Below this value, when $Q_{e}$ is larger or $A_{h}$ is smaller, the density current evolves unstably.

To estimate how this critical $E_{h}$ value corresponds to a real coastal area, we briefly consider the situation in the East China Sea coastal areas. The density current in the East China Sea flows $700 \mathrm{~km}$ in length, extending from the mouth of Yangtze River to the Taiwan Strait, and is mainly induced by fresh water discharges from the Yangtze River. According to Beardsley et al. [1985], peak discharge of the Yangtze River $\left(45 \times 10^{3} \mathrm{~m}^{3} \mathrm{~s}^{-1}\right)$ usually occurs from late June to August and minimum discharge $\left(10 \times 10^{3} \mathrm{~m}^{3} \mathrm{~s}^{-1}\right)$ occurs in January and February. Thus our numerical result indicates that when $A_{h}$ in the coastal area is about $8.5 \times 10^{5} \mathrm{~cm}^{2} \mathrm{~s}^{-1}$, the density current will be unstable in all seasons. However, if $A_{h}$ exceeds $2.3 \times 10^{6} \mathrm{~cm}^{2} \mathrm{~s}^{-1}$, the density current will develop unstably only in the summer peak discharge period.

In cases where $E_{h}$ is smaller than the critical value, the numerical experiments showed that excessive accumulation of fresh water near the upper coast promotes the vertical advection of lower layer saline water, inevitably causing sudden reversals in the time change of the salinity near the coast. Such temporal reversals in the density field break down the quasisteady balance of the density currents and promote grid-scale disturbances in the longshore direction. After the vertical shear of the density current exceeds the threshold value of the instability, the grid-scale disturbances evolve rapidly into large-scale unstable waves through the nonlinear cascading process. In the numerical experiments, the linear growth rate of the unstable waves ranges between 2 and 7 days in the $e$-folding time scale and the unstable eddy scales range between 50 and $100 \mathrm{~km}$.

As was mentioned in the introduction, the scale of eddies observed on density currents in the real ocean is of the order of $50-100 \mathrm{~km}$, which corresponds well to that in the numerical experiments. The growth rate of unstable waves in the real ocean, however, is rather difficult to ascertain from a single synoptic observation. Griffiths and Pearce [1985] reported that in the Leeuwin Current, small perturbations took 10-15 days to become well-developed eddies. In the unstable case considered in section 3 , the growth from small perturbations to fully developed eddies can be seen between days 33 and 43 (compare Figure $6 a$ ). This time interval is in the same range as that observed by Griffiths and Pearce [1985].

Parametric studies in the numerical experiments further revealed that the unstable waves' linear growth rate decreases with increasing $E_{h}$, while the unstable eddy scale increases as $E_{h}$ increases. This $E_{h}$ dependency and the existence of the short wave cutoff and the longwave cutoff in the numerical results can be qualitatively explained by the two-layer quasigeostrophic instability theory with the horizontal eddy diffusivity effect included. Holland and Schmitz [1985] also found that a large $A_{h}$ (or $E_{h}$ ) value decreases the growth rate of the most unstable wave in a two-level quasi-geostrophic model, in which they considered a horizontally sheared jet resembling the Gulf Stream.

Study of the energetics of unstable waves revealed that at the early stages of instability, perturbations grow by subtracting energy from the available potential energy of the density current, that is, instability occurs via the baroclinic process. This result suggests that the motion in the finite-depth lower layer is crucial in determining the instability of the coastal density currents.

As in the laboratory experiments of Griffiths and Linden [1981, 1982] and in the field observations of Griffiths and Pearce [1985], we also observed in our numerical experiments cyclonic-anticyclonic eddy pairs and backward breaking waves on the unstable density currents. In order to examine the detachment process of the cyclonic-anticyclonic eddies at the nonlinear stage, we tracked passive particles in the surface level. After the density current becomes unstable, we found that low-salinity water in the density current is "blocked" offshore owing to the anticyclonic eddies advancing seaward. At the same time, as cyclonic eddies develop near the coast, outer oceanic water is effectively entrained shoreward. The movement of this outer oceanic water toward the coast exhibits a streamerlike pattern and is similar to the surface temperature patterns observed from satellite pictures [e.g., Griffiths and Pearce, 1985].

After the unstable waves attain large amplitudes, we found that an energy transfer from the EKE to the MKE field exists through Reynolds stresses. In the model basin, this energy feedback accelerates the density current near the coast and stimulates a barotropic flow offshore moving with the coast to the left. These induced mean flows subsequently cut off the fully developed unstable waves and advect the cyclonic (anticyclonic) eddies in the downstream (upstream) direction with a speed of $10 \mathrm{~cm} \mathrm{~s}^{-1}$. On the Leeuwin Current, Cresswell [1977] observed an anticyclonic eddy which was stationary for the first 30 days and then started moving upstream at $5 \mathrm{~cm} \mathrm{~s}^{-1}$. This movement of the anticyclonic eddy may be caused by the above mechanisms of our numerical experiments.

Acknowledgments. The authors wish to express their thanks to $\mathrm{H}$. Kunishi of Kyoto University for his encouragement throughout this study. They are also grateful to A. Kubokawa of Tohoku University for his valuable comments and critical reading of the manuscript. Thanks are extended to other members at the Geophysical Institute of Kyoto University for their helpful discussions. The computations were performed on the FACOM M382 and VP-100 computer at the Data Processing Center of Kyoto University. A part of this study was supported by grant-in-aid 60420014 for scientific research in 1985 from the Ministry of Education of Japan.

\section{REFERENCES}

Awaji, T., N. Imasato, and H. Kunishi, Tidal exchange through a strait: A numerical experiment using a simple model basin, $J$. Phys. Oceanogr., 10, 1499-1508, 1980.

Beardsley, R. C., R. Limeburner, H. Yu, and G. A. Cannon, Discharge of the Changjiang (Yangtze River) into the East China Sea, Cont. Shelf Res., 4, 57-76, 1985.

Crepon, M., L. Wald, and J. M. Monget, Low-frequency waves in the Ligurian Sea during December 1977, J. Geophys. Res., 87, 595-600, 1982.

Cresswell, G. R., The trapping of two drifting buoys by an ocean eddy, Deep Sea Res., 24, 1203-1209, 1977.

Cresswell, G. R., and T. G. Golding, Observations of south-flowing current in the southeastern Indian Ocean, Deep Sea Res., 27, 449$466,1980$.

Grifliths, R. W., and E. J. Hopfinger, Gravity currents moving along a lateral boundary in a rotating fluid, J. Fluid Mech., 134, 357-399, 1983.

Griffiths, R. W., and P. F. Linden, The stability of buoyancy driven coastal currents, Dyn. Atmos. Oceans, 5, 281-306, 1981.

Grifliths, R. W., and P. F. Linden, Laboratory experiments on fronts, 1, Density-driven boundary currents, Geophys. Astrophys. Fluid Dyn., 19, 159-187, 1982. 
Griffiths, R. W., and A. F. Pearce, Instability and eddy pairs on the Leeuwin Current south of Australia, Deep Sea Res., 32, 1511-1534, 1985.

Holland, W. R., and W. J. Schimitz, Zonal penetration scale of model midlatitude jets, J. Phys. Oceanogr., 15, 1859-1875, 1985.

Ikeda, M., Coastal flows driven by a local density flux, J. Geophys. Res., 89, 8008-8016, 1984.

Imasato, N., T. Awaji, and $\mathrm{H}$. Kunishi, Tidal exchange through Naruto, Akashi and Kitan Straits, J. Oceanogr. Soc. Jpn., 36, 151$162,1980$.

Kao, T. W., The dynamics of oceanic fronts, I, The Gulf Stream, $J$. Phys. Oceanogr., 10, 483-492, 1980.

Kao, T. W., The dynamics of oceanic fronts, II: Shelf water structure due to freshwater discharges, $J$. Phys. Oceanogr., 11, 1215-1223, 1981.

Killworth, P. D., and M. E. Stern, Instabilities on density-driven boundary currents and fronts, Geophys. Astrophys. Fluid Dyn., 22, $1-28,1982$

Killworth, P. D., N. Paldor, and M. E. Stern, Wave propagation and growth on a surface front in a two-layer geostrophic current, $J$. Mar. Res., 42, 761-785, 1984.

Kubokawa, A., Instability caused by the coalescence of two modes of a one-layer coastal current with a surface front, J. Oceanogr. Soc. Jpn., 42, 373-380, 1986.

Kubokawa, A., and K. Hanawa, A theory of semigeostrophic gravity waves and its application to the intrusion of a density current along a coast, I, Semigeostrophic gravity waves, J. Oceanogr. Soc. Jpn., 40, 247-259, 1984.

Lee, T. N., and L. P. Atkinson, Low-frequency current and temperature variability from Gulf Stream frontal eddies and atmospheric forcing along the southeast U.S. outer continental shelf, $J$. Geophys. Res., 88, 4541-4567, 1983.

Legeckis, R., and G. Cresswell, Satellite observations of sea surface temperature fronts off the coast of western and southern Australia, Deep Sea Res., 28, 279-306, 1981.
Mork, M., Circulation phenomena and frontal dynamics of the Norwegian Coastal Current, Philos. Trans. R. Soc. London, 302, 635$647,1981$.

Orlanski, I., and M. D. Cox, Baroclinic instability in ocean currents, Geophys. Fluid Dyn., 4, 297-332, 1973.

Paldor, N., Stability and stable modes of coastal fronts, Geophys. Astrophys. Fluid Dyn., 27, 217-228, 1983.

Pedlosky, J., Geophysical Fluid Dynamics, 624 pp., Springer, New York, 1979.

Pratt, L. J., and M. E. Stern, Dynamics of potential vorticity fronts and eddy detachment, J. Phys. Oceanogr., 16, 1101-1120, 1986.

Semtner, A. J., An oceanic general circulation model with bottom topography, Numerical simulation of weather and climate, Tech. Rep. 9, Dep. of Meteorol., Univ. of Calif., Los Angeles, 1974.

Stern, M. E., Geostrophic fronts, bores, breaking and blocking waves, J. Fluid Mech., 99, 687-703, 1980.

Stern, M. E., Large-scale lateral wave breaking and shingle formation, J. Phys. Oceanogr., 15, 1274-1283, 1985.

Stern, M. E., J. A. Whitehead, and B. L. Hua, The intrusion of a density current along the coast of a rotating fluid, J. Fluid Mech., 123, 237-266, 1982.

Zhang, Q. A., and V. Klemas, Determination of winter temperature patterns, fronts, and surface current in the Yellow Sea and East China Sea from satellite imagery, Remote Sens. Environ., 12, 201218,1982 .

T. Awaji, Department of Applied Science, Kochi Women's University, Kochi 780, Japan.

N. Imasato and B. Qiu, Geophysical Institute, Kyoto University, Kyoto 606, Japan. 